\title{
Phytochemistry, pharmacological activities and traditional uses of Emblica officinalis: A review
}

\author{
${ }^{*}$ Md. Rubaiyat Hasan, Md. Nasirul Islam and Md. Rokibul Islam \\ Department of Biotechnology and Genetic Engineering, Islamic University, Kushtia-7003, Bangladesh
}

\begin{abstract}
From the ancient time, plants have been playing a key role for the betterment of mankind presenting as an extraordinary source of natural medicine. The complexity in formulating chemical based drugs as well as their health related side effects and uprising cost has led worldwide researchers to focus on medicinal plant research. Bangladesh has a vast repository of diverse plant species where about five thousand plants species have been claimed as having significant medicinal values. The researched papers on medicinal plants publishing from last few decades mention the activities of different plant bioactive compounds that are used widely in the treatment of various human ailments. Emblica officinalis is reported to possess bioactive compounds like tannins, flavonoids, saponins, terpenoids, ascorbic acids and many other compounds which are confirmed to have diverse pharmacological activities like antimicrobial, antioxidant, anti-inflammatory, radio-protective, hepatoprotective, antitissuive, immunomodulatory, hypolipedemic and many other activities. This medicinal plant is also reported to have anticancer, anti HIV-reverse transcriptase, antidiabetic, antidepressant, antiulcerogenic, wound healing activities and so forth. The current review paper summarizes the phytochemical constituents, pharmacological activities and traditional uses of the plant Emblica officinalis.
\end{abstract}

Key Words: Euphorbiaceae, Amla, Bangladesh.

\section{INTRODUCTION}

Emblica officinalis Gaertn. (Family-Euphorbiaceae) also known as Phyllanthus emblica, is commonly known as 'Amla' or 'amlaki' in Bengali and 'Indian gooseberry' in English. This species is medium sized deciduous tree with 8-18 meters height and is native to tropical southeastern Asia, particularly in central and southern India, Pakistan, Bangladesh, Sri Lanka, southern China, the Mascarene Islands and Malaysia (Table 1). In India, Amla trees are found throughout the forests of tropical area ascending up to $4500 \mathrm{ft}$ on hills (Rai et al., 2012; Thilaga et al., 2013). Amla is rich in fiber, carbohydrate, iron and is reported as the richest source of vitamin C (Singh et al., 2011) (Table 2). The fruit is also used in a combination form known as Triphala meaning three fruits which is a Thai traditional herbal formulation composed of Emblica officinalis, Terminalia belerica and Terminalia chebula (Phetkate et al., 2012).

Many herbal and patent drugs have been formulated by the constituents of this plant (Rai et al., 2012). E. officinalis primarily contains tannins, flavonoids, phenolic compounds, saponins, terpenoids, ascorbic acids, carbohydrates and many other compounds (Khan, 2009). Supplements of fresh amla fruit is very favorable to individuals suffering from anemia. The juice of fresh amla fruit is given as diuretic, anti-bilious remedy and as a tonic. It is also helpful in over thirst, dyspepsia, burning sensation and other complaints of digestive system (Kumar et al., 2012b).

\section{Taxonomy}

Taxonomical classification of E. officinalis is summarized in table 3.
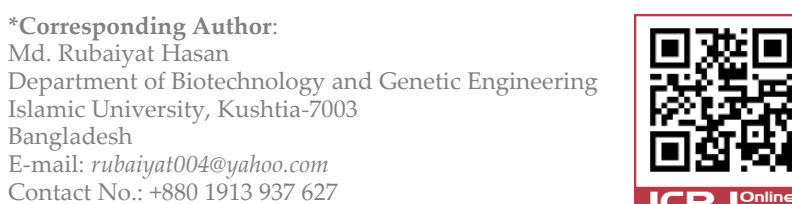

\section{PHYTOCHEMISTRY}

This herb has many bioactive compounds including apigenin, gallic acid, ellagic acid, chebulinic acid, quercetin, chebulagic acid, corilagin, isostrictiniin, methyl gallate, luteolin and so on. Emblicanin A, emblicanin B, phyllaemblicin B, punigluconin and pedunculagin are tannins present in Emblica officinalis (Table 4). Glutamic acid, proline, aspartic acid, alanine, and lysine are $29.6 \%$, $14.6 \%, 8.1 \%, 5.4 \%$ and $5.3 \%$ respectively of the total amino acids. The pulpy portion of fruit, dried and freed from the nuts contains: gallic acid $1.32 \%$, tannin, gum $13.75 \%$; albumin $13.08 \%$; crude cellulose $17.08 \%$; mineral matter $4.12 \%$ and moisture $3.83 \%$. Amla fruit ash contains chromium, 2.5 ppm; zinc 4 ppm; and copper, 3 ppm (Kumar et al., 2012a). Nickel and lead metals were not found in leaves of Emblica officinalis. The level of copper was found higher in the sample leaves of Emblica officinalis followed by chromium, manganese and zinc (Kumar et al., 2013). Chemical constituents from different plant parts of are illustrated below:

Leaves: It contains gallic acid, chebulic acid, ellagic acid, chebulinic acid, chebulagic acid, amlic acid, alkaloids phyllantine and phyllantidine (Khan, 2009).

Seeds: A fixed oil, phosphatides and a small quantity of essential oil. The fixed oil (acid value 12.7; saponification value 185; iodine value 139.5; acetyl value 2.03; unsaponifiable matter $3.81 \%$; sterol $2.70 \%$; saturated fatty acid $7 \%$. Contains linolenic acid $(8.78 \%)$, linoleic $(44 \%)$. oleic $(28.40 \%)$, steric $(2.15 \%)$, palmitic $(2.99 \%)$ and miristic acid $(0.95 \%)$ (Khan, 2009).

Barks: Contain leukodelphinidin, tannin and proanthocyanidin (Khan, 2009).

Roots: Contain ellagic acid and lupeol (Khan, 2009). 
Table 1: Botanical description of E. officinalis.

\begin{tabular}{|c|c|c|}
\hline Feature & Description & Reference \\
\hline Habitat & $\begin{array}{l}\text { Central and southern India, Pakistan, Bangladesh, Sri Lanka, Malaysia, southern China, the } \\
\text { Mascarene Islands, South East Asia and Uzbekistan. }\end{array}$ & $\begin{array}{l}\text { Rai et al., 2012; } \\
\text { Thilaga et al., 2013; } \\
\text { Khan, } 2009\end{array}$ \\
\hline Appearance & $\begin{array}{l}\text { Medium sized deciduous tree, } 8-18 \text { meters height with thin light grey bark exfoliating in small } \\
\text { thin irregular flakes. }\end{array}$ & Meena et al., 2010 \\
\hline Used parts & Dried fruits, fresh fruit, seed, leaves, root bark, flowers. & $\begin{array}{l}\text { Khan, 2009; Kumar et } \\
\text { al., 2012b }\end{array}$ \\
\hline Leaves & $\begin{array}{l}\text { Simple, sub sessile, closely set along the branchlets, light green having the appearance of } \\
\text { pinnate leaves. }\end{array}$ & Meena et al., 2010 \\
\hline \multirow[t]{4}{*}{ Fruits } & $\begin{array}{l}15-20 \mathrm{~mm} \text { long and } 18-25 \mathrm{~mm} \text { wide, nearly spherical or globular wider than long and with a } \\
\text { small and slight conic depression on both apexes. Mesocarp is yellow and endocarp is } \\
\text { yellowish brown in ripened condition }\end{array}$ & Khan, 2009 \\
\hline & $\begin{array}{l}\text { Globose, fleshy, pale yellow with six obscure vertical furrows enclosing six trigonous seeds in } \\
\text { 2-seeded } 3 \text { crustaceous cocci. }\end{array}$ & Meena et al., 2010 \\
\hline & $\begin{array}{l}\text { Seedlings start bearing fruits in 7-8 years after planting, while the budded clones will start } \\
\text { bearing fruits from the } 5 \text { th year onwards. }\end{array}$ & Kumar et al., 2012a \\
\hline & $\begin{array}{l}\text { Fresh fruits are light green and ripe fruits turn light brown in colour. The average weight of the } \\
\text { fruit is } 60-70 \mathrm{~g} \text {. }\end{array}$ & Kumar et al., 2012b \\
\hline Flowers & $\begin{array}{l}\text { Greenish yellow, in axillary fascicles, unisexual, males numerous on short slender pedicels, } \\
\text { females few, sub sessile, ovary 3-celled. }\end{array}$ & $\begin{array}{l}\text { Meena et al., 2010; } \\
\text { Rai } \text { et al., } 2012\end{array}$ \\
\hline Seeds & Four-Six, smooth, dark brown & Khan, 2009 \\
\hline Barks & Thick to $12 \mathrm{~mm}$, shining grayish brown or grayish green & Khan, 2009 \\
\hline $\begin{array}{l}\text { Flowering } \\
\text { and fruiting }\end{array}$ & February - May and December - January & Rai et al., 2012 \\
\hline Edible part & Mesocarp and endocarp that forms the hard stone which encages the seed & Patel and Goyal, 2011 \\
\hline
\end{tabular}

\section{PHARMACOLOGICAL INVESTIGATIONS}

\section{Antibacterial activity}

Antibacterial activities of different solvent extracts and isolated compounds from Emblica officinalis are shown in table 5.

\section{Antifungal activity}

Antifungal property of E. officinalis was reported against Aspergillus (Satish et al., 2007). Fruit ethanol and acetone extracts showed moderate activity against Fusarium equiseti and Candida albicans where Grisofulvin was used as standard antibiotic (Hossain et al., 2012). Plant methanolic extract of E. officinalis did not show antifungal activity against phytopathogenic fungi Aspergillus niger F2723 (Bobbarala et al., 2009).

\section{Antioxidant and free radical scavenging activity}

Galic acid equivalent as total phenolic content from fruit and seed of E. officinalis has excellent antioxidant properties and play an important role as free radical scavengers required in the maintenance of "redox homeostasis" responsible for diverse degenerative diseases (Prakash et al., 2012). The methanolic seed extract of Emblica officinalis has promising free radical scavenging activity of 1,1 , Diphenyl-2-picryl-hydrazil (DPPH) in a concentration dependant manner (Priya et al., 2012). Methanolic extract of fruit pulp also have antioxidant and free radical scavenging activity (Mehrotra et al., 2011; Liu et al., 2008a; Liu et al., 2008b, Hazra et al., 2010, Majumdar et al., 2010).
Methanolic extracts of dried leaves of Phyllanthus emblica was used for the comparative study of antibacterial and antioxidant activity and the research work was ended positively showing the extract has both these activities (Shivaji et al., 2010). In a separate research work, it is seen that the water extract of E. officinalis fruit prepared according to Thai Herbal Pharmacopoeia has a strong potential for free radical scavenging, ferric reducing as well as inhibiting ROS (reactive oxygen species) production (Charoenteeraboon et al., 2010).

\section{Insecticidal activity}

Saponins which are important constituents of E. officinalis have insecticidal or cytotoxic properties to certain insects (Chaieb, 2010). Although saponins which had shown insecticidal activity was collected from natural sources other than E. officinalis. But as saponins are bioactive compounds found in E. officinalis too, it is obvious that $E$. officinalis might have insecticidal activity and further evaluation can be conducted to get more precise evaluation.

\section{Larvicidal and mosquitocidal activity}

In a mosquitocidal property evaluation test Murugan et al. (2012) observed larvicidal and pupicidal activities of methanol extract of E. officinalis against the malarial vector, Anopheles stephensi showing $98 \%$ mortality rate at $100 \mathrm{ppm}$. The ethanol and methanol extracts of E. officinalis also exerted $100 \%$ mortality (no hatchability) at $400 \mathrm{ppm}$ and above (Murugan et al., 2012). Jeyasankar et al. (2012) 
Table 2: Nutritional value of Emblica officinalis (redrawn from Singh et al., 2011).

\begin{tabular}{ll}
\hline Chemical components & Percentage \\
\hline Fruits: Moisture & $81.2 \%$ \\
Protein & $0.5 \%$ \\
Fat & $0.1 \%$ \\
Mineral matter & $0.7 \%$ \\
Fiber & $3.4 \%$ \\
Carbohydrate & $14.1 \%$ \\
Bulk elements $\mathrm{Mg} / 100 \mathrm{~g}$ & Net weight \\
Calcium & $0.05 \%$ \\
Phosphorus & $0.02 \%$ \\
Iron & $1.2 \mathrm{mg} / 100 \mathrm{~g}$ \\
Vitamin C & $600 \mathrm{mg} / 100 \mathrm{~g}$ \\
Nicotinic acid & $0.2 \mathrm{mg} / 100 \mathrm{~g}$ \\
\hline
\end{tabular}

Table 3: Taxonomical classification of E. officinalis.

\begin{tabular}{|ll|}
\hline Kingdom & Plantae (Plants) \\
Subkingdom & Tracheobionta (Vascular plants) \\
Superdivision & Spermatophyta (Seed plants) \\
Division & Angiospermae (Flowering plants) \\
Class & Dicotyledonae (Dicotyledons) \\
Subclass & Rosidae \\
Order & Geraniales \\
Family & Euphorbiaceae \\
Genus & Emblica \\
Species & officinalis Geartn. \\
\hline
\end{tabular}

reported that the larvicidal activity of Phyllanthus emblica ethyl acetate leaf extracts. The study concluded that the ethyl acetate extract of $P$. emblica exhibited the maximum larvicidal activity $(99.6 \%)$ with LC50 (lethal Concentration brings out $50 \%$ mortality) value of $78.89 \mathrm{ppm}$ against the larvae of Aedes aegypti (Jeyasankar et al., 2012).

\section{Antidepressant activity}

Pemminati et al. (2010) has checked the antidepressant activity of aqueous extract of fruits of E. officinalis in inbred adult male Swiss Albino mice weighing 25-30g. The test was carried out by forced swim test (FST) and tail suspension test (TST). The result of this test showed the antidepressant activity of E. officinalis as comparable to the of standard antidepressant drug imipramine.

\section{Immunomodulatory activity}

Reports suggest that triphala can stimulate the neutrophil functions in the immunized albino rats (Srikumar et al., 2005). There was considerable dose dependent raise in haemagglutination antibody titre, macrophage migration index, hypersensitivity reaction, respiratory burst activity of the peritoneal macrophages, total leukocyte count, percentage lymphocyte distribution, serum globulin and relative lymphoid organ weight in Emblica treated albino mice indicating its ability to stimulate humoral and cell mediated immunity along with macrophage phagocyte (Suja et al., 2009).

\section{Anti-inflammatory activity}

E. officinalis showed anti-inflammatory activities in carrageenan induced acute and cotton pellet induced chronic inflammation in Sprague-Dawley rats by reducing paw volume in acute inflammation and by decreasing cotton pellet induced granulomas tissue lipid peroxidation, the granulomatous tissue mass, myeloperoxidase activity and plasma extravasation in chronic inflammato- ry condition (Muthuraman et al., 2011). E. officinalis water extract has reported to have inhibitory effect on the synthesis and release of inflammatory mediators in rats (Jaijoy et al., 2010).

\section{Radioprotective activity}

It has been reported that mice treated with Emblica officinalis extract before exposure to different doses of gamma radiation can reduce the severity of symptoms of radiation sickness and mortality (Singh et al., 2006). Similar delayed onset of mortality and reduction in the symptoms of radiation sickness in mice were seen in consecutively triphala treated mice before irradiation when compared with the non-drug treated irradiated controls (Jagetia et al., 2002).

\section{Hypolipidemic activity}

Amla fruit have been reported to have significant antihyperlipidemic, hypolipidemic, and anti-atherogenic effect (Santoshkumar et al., 2013). Treatment with Emblica officinalis caused significant reduction of Total Cholesterol (TC), Low Density Lipoprotein (LDL), triglyceride (TG) and Very Low Density Lipoprotein (VLDL), and a significant increase in High Density Lipoprotein (HDL) levels in patients with type II hyperlipidemia. Both treatments from E. officinalis and simvastatin produced significant reduction in blood pressure; however, this beneficial effect was more marked in patients receiving $E$. officinalis (Gopa et al., 2012). Histopathological study of thoracic aorta of Emblica officinalis treated group has shown decrease in atherogenicity compared to untreated high cholesterol diet fed rats. The data demonstrated that Emblica officinalis formulation was associated with hypolipidemic effects on the experimentally induced hypercholesteremic rats (Kumar and Kalaivani, 2011). It is also seen that E. officinalis treated rat showed more hypoglycemic and hypolipidemic activity than Phyllanthus acidus treated diabetic rats (Modilal and Pitchai, 2011).

\section{Cytotoxic effects}

To evaluate the immunostimulatory and side effects of Triphala in a clinical phase I, all the volunteers took Triphala for two weeks (3 capsules per day). As complete physical examinations, routine laboratory analysis and immunological studies were performed before ingestion and after initial meeting for 4 consecutive weeks. The result revealed significant immunostimulatory effects on cytotoxic T cells (CD3-CD8+) and natural killer cells (CD16+CD56+). Both of them increased significantly when compared with those of the control samples. However, no significant change in cytokine secretion was detected. All volunteers were healthy and showed no adverse effects throughout the duration of the study (Phetkate et al., 2012). Flavonoids, a group of essential bioactive secondary metabolites of Emblica officinalis, were evaluated for antioxidant potential, cytotoxicity and intestinal absorption. The research concluded that flavonoids from $E$. officinalis and some other medicinal plants hold a good prospective as nutraceutical \& chemotherapeutics agents because of their antioxidant potential, no cytotoxicity and good intestinal absorptive property (Sharma et al., 2010). But it is confirmed that the chloroform soluble fraction of the ripe fruits of Amlaki containing alkaloids have both antimicrobial and cytotoxic activity (Rahman et al., 2009).

Anti-diabetic and hypoglycemic activity

Herbal formulations prepared by extracts of Tinospora cordifolia, Trigonella foenum and Emblica officinalis were 
Table 4: Properties, functions and some common sources of bioactive compounds isolated from E. officinalis.

\begin{tabular}{|c|c|c|c|c|c|c|}
\hline $\begin{array}{l}\text { Compound } \\
\text { names }\end{array}$ & $\begin{array}{l}\text { Molecular } \\
\text { formula }\end{array}$ & $\begin{array}{l}\text { Molecular } \\
\text { weight }\end{array}$ & $\mathrm{BP} / \mathrm{MP}$ & Biological activity & Common sources & References \\
\hline $\begin{array}{l}\text { Chebulinic } \\
\text { acid }\end{array}$ & $\mathrm{C}_{41} \mathrm{H}_{32} \mathrm{O}_{27}$ & $\begin{array}{l}956.67 \\
\mathrm{gm} / \mathrm{mol}\end{array}$ & $\begin{array}{c}1460^{\circ} \mathrm{C} \text { at } 760 \\
\mathrm{mmHg}(\mathrm{BP})\end{array}$ & $\begin{array}{l}\text { Antioxidant activity, Anti- } \\
\text { secretory and cryo-protective } \\
\text { activity }\end{array}$ & $\begin{array}{l}\text { Phyllanthus emblica, } \\
\text { Terminalia arborea, and T. } \\
\text { chebula }\end{array}$ & $\begin{array}{l}\text { Baliga and } \\
\text { Dsouza, 2010; } \\
\text { Mishra, 2013 }\end{array}$ \\
\hline $\begin{array}{l}\text { Chebulagic } \\
\text { acid }\end{array}$ & $\mathrm{C}_{41} \mathrm{H}_{30} \mathrm{O}_{27}$ & $\begin{array}{l}954.66 \\
\mathrm{gm} / \mathrm{mol}\end{array}$ & $\begin{array}{l}1610.6^{\circ} \mathrm{C} \text { at } \\
760 \mathrm{mmHg} \\
\text { (BP) }\end{array}$ & Antispasmodic action & $\begin{array}{l}\text { E. officinalis, Terminalia } \\
\text { Chebula, T. citrine, T. } \\
\text { catappa }\end{array}$ & $\begin{array}{l}\text { Reddy et al., } 2009 \\
\text { Chen and Li, } 2006\end{array}$ \\
\hline Emblicanin-A & $\mathrm{C}_{34} \mathrm{H}_{22} \mathrm{O}_{22}$ & $\begin{array}{l}<1,000 \\
\mathrm{gm} / \mathrm{mol}\end{array}$ & $\begin{array}{l}\text { Not con- } \\
\text { firmed }\end{array}$ & Antioxidant activity & E. officinalis & $\begin{array}{l}\text { Madhuri et al., } \\
2011\end{array}$ \\
\hline Emblicanin-B & $\mathrm{C}_{34} \mathrm{H}_{22} \mathrm{O}_{22}$ & $\begin{array}{l}<1,000 \\
\mathrm{gm} / \mathrm{mol}\end{array}$ & $\begin{array}{l}\text { Not con- } \\
\text { firmed }\end{array}$ & Antioxidant activity & E. officinalis & $\begin{array}{l}\text { Madhuri et al., } \\
2011\end{array}$ \\
\hline Gallic acid & $\mathrm{C}_{7} \mathrm{H}_{6} \mathrm{O}_{5}$ & $\begin{array}{l}170.12 \\
\mathrm{gm} / \mathrm{mol}\end{array}$ & $252^{\circ} \mathrm{C}(\mathrm{MP})$ & $\begin{array}{l}\text { Radioprotective effect, chemopre- } \\
\text { ventive effect, anti-carcinogenic, } \\
\text { antioxidative, antimutagenic, anti- } \\
\text { allergic and anti-inflammatory } \\
\text { activities. }\end{array}$ & $\begin{array}{l}\text { E. officinalis; T chebula; } T \\
\text { bellerica, } C \text { sinensis L., } \\
\text { Arctostaphylos uva-ursiL., } \\
\text { C avellana, O biennis, } V \\
\text { viniferaL. }\end{array}$ & $\begin{array}{l}\text { Baliga and } \\
\text { Dsouza, 2010; } \\
\text { Vazirian et al., } \\
\text { 2011; Negi et al., } \\
\text { 2005; Karamaæ et } \\
\text { al., 2006 }\end{array}$ \\
\hline Ellagic acid & $\mathrm{C}_{14} \mathrm{H}_{6} \mathrm{O}_{8}$ & $\begin{array}{c}302 \\
\mathrm{gm} / \mathrm{mol}\end{array}$ & $\geq 350{ }^{\circ} \mathrm{C}(\mathrm{MP})$ & $\begin{array}{l}\text { Radioprotective and chemopreven- } \\
\text { tive effect, antityrosinase Activity, } \\
\text { antioxidant, antiproliferative, and } \\
\text { antiatherogenic Properties, } \\
\text { estrogenic/antiestrogenic Activity }\end{array}$ & $\begin{array}{l}\text { E. officinalis, Castanea } \\
\text { sativa, Euca-lyptus } \\
\text { camaldulensis, Juglans } \\
\text { regia }\end{array}$ & $\begin{array}{l}\text { Baliga and } \\
\text { Dsouza, 2010; } \\
\text { O"zer et al., 2007; } \\
\text { Papoutsi } \text { et al., } \\
2005\end{array}$ \\
\hline Quercetin & $\mathrm{C}_{15} \mathrm{H}_{10} \mathrm{O}_{7}$ & $\begin{array}{l}302.24 \\
\mathrm{gm} / \mathrm{mol}\end{array}$ & $316.5^{\circ} \mathrm{C}(\mathrm{MP})$ & $\begin{array}{l}\text { Radioprotective, chemopreventive, } \\
\text { hepato protective effect }\end{array}$ & E. officinalis & $\begin{array}{l}\text { Baliga and } \\
\text { Dsouza, 2010; } \\
\text { Madhuri et al., } \\
2011\end{array}$ \\
\hline Phyllantine & $\mathrm{C}_{14} \mathrm{H}_{17} \mathrm{NO}_{3}$ & $\begin{array}{l}247.29 \\
\mathrm{gm} / \mathrm{mol}\end{array}$ & $\begin{array}{l}\text { Not con- } \\
\text { firmed }\end{array}$ & Not confirmed & E. officinalis & Khan, 2009 \\
\hline Phyllantidine & $\mathrm{C}_{13} \mathrm{H}_{15} \mathrm{NO}_{3}$ & $\begin{array}{l}233.2631 \\
\mathrm{gm} / \mathrm{mol}\end{array}$ & $\begin{array}{l}\text { Not con- } \\
\text { firmed }\end{array}$ & $\begin{array}{l}\text { Neuropharmacological activity } \\
\text { (CNS activity) }\end{array}$ & $\begin{array}{l}\text { E. officinalis, P. discoides; } \\
\text { Seurinega suffruticosa }\end{array}$ & $\begin{array}{l}\text { Khan, 2009; } \\
\text { Beutler et al., } 1985\end{array}$ \\
\hline Punigluconin & $\mathrm{C}_{34} \mathrm{H}_{26} \mathrm{O}_{23}$ & $\begin{array}{l}802.556 \\
\mathrm{gm} / \mathrm{mol}\end{array}$ & $\begin{array}{l}1448.6^{\circ} \mathrm{C} \text { at } \\
760 \mathrm{mmHg} \\
\text { (BP) }\end{array}$ & Antioxidant activity & E. officinalis & $\begin{array}{l}\text { Bhattacharya et al. } \\
1999\end{array}$ \\
\hline Pedunculagin & $\mathrm{C}_{34} \mathrm{H}_{24} \mathrm{O}_{22}$ & $\begin{array}{l}784.54 \\
\mathrm{gm} / \mathrm{mol}\end{array}$ & $\begin{array}{c}1578.039^{\circ} \mathrm{C} \\
\text { at } 760 \mathrm{mmHg} \\
(\mathrm{BP})\end{array}$ & $\begin{array}{l}\text { Antitumor activity, Antioxidant } \\
\text { activity }\end{array}$ & E. officinalis & $\begin{array}{l}\text { Chang et al., 1995; } \\
\text { Bhattacharya et al. } \\
1999\end{array}$ \\
\hline
\end{tabular}

evaluated for hypoglycemic effects and Oral Glucose Tolerance Test (OGTT) in normal and Alloxan induced diabetic rats and significant, marginal and very less decrease in blood glucose level was observed when different herbal combinations were used (Deep et al., 2011).

The polyherbal combination of extracts E. officinalis (fruit), Momordica charantia (fruit) and Trigonella foenumgraecum (leaves and seeds) had shown synergistic activity, as the glucose levels were decreased more significantly by the combination of extracts compared to the individual extract when used separately in streptozotocin induced diabetic rats (Satyanarayana et al., 2010). The aqueous fruit extract of Phyllanthus emblica was evaluated on type-II diabetes, triglycerides (TG) and liver-specific enzyme, alanine transaminase (ALT). This study showed that in a dose of $200 \mathrm{mg} / \mathrm{kg}$ body weight the aqueous fruit extract can significantly reduce the blood glucose level in alloxaninduced diabetic rats (Qureshi et al., 2009). Another study reports that Phyllanthus emblica treated rat showed more hypoglycemic and hypo lipidemic activity than Phyllanthus acidus treated diabetic rats when the effect of orally administered aqueous extracts ( $350 \mathrm{mg} / \mathrm{kg}$ body weight) of fruits of Phyllanthus emblica and Phyllanthus acidus on serum glucose, glycosylated hemoglobin, insulin, cholesterol, triglycerides, HDL-cholesterol, protein, urea and creatinine were examined in control and extracttreated diabetic rats (Modilal and Pitchai, 2011).

\section{Hepato-protective activity}

The histopathological study of liver cells of rats was examined by administering E. officinalis as a preventative agent to reduce paracetamol induced hepatotoxicity and it has been observed that fruit extract has the ability to rectify toxicity or hepatic damage (Malar and Bai, 2009). Another histological study was undertaken to demonstrate the protective effect of $50 \%$ hydroalcoholic extract of the fresh fruit of E. officinalis against chronic toxicity induced by carbon tetrachloride and thioacetamide in rats. From the liver sections of the tested rats, it was observed that E. officinalis reversed the abnormal histopathology by accelerating the regenerative activity and in a few cases, the hepatocytic injury was found negligible in E. officinalis treated group of rats (Mir et al., 2007).

\section{Anti-cancer and anti-proliferative activity}

E. officinalis exhibits its anticancer activities through inhibition of activator protein-1 and targets transcription of viral oncogenes responsible for development of cervical 
Table 5: Antibacterial activity of deferent solvent extracts and isolated compounds from Emblica officinalis.

\begin{tabular}{|c|c|c|c|c|c|c|}
\hline $\begin{array}{l}\text { Used extracts/ } \\
\text { other compounds }\end{array}$ & Used organisms & Extract conc. & $\begin{array}{l}\text { Max. zone of } \\
\text { inhibition } \\
(\mathrm{mm})\end{array}$ & $\begin{array}{l}\text { Organism(s) } \\
\text { showed highest } \\
\text { activity }\end{array}$ & $\begin{array}{l}\text { Extract or extract } \\
\text { conc. showed } \\
\text { highest activity }\end{array}$ & Reference \\
\hline $\begin{array}{l}\text { Ethanol, Acetone } \\
\text { (Fruit extract) }\end{array}$ & $\begin{array}{l}\text { V. cholerae, S. aureus, } P \text {. } \\
\text { aeruginasa, } \\
\text { B. subtilis, Shigella dysenteriae, } \\
\text { S. pyogenous, E. coli, } \\
\text { B. megaterium }\end{array}$ & $0.5 \mathrm{mg} / \mathrm{disc}$ & 12.7 & Shigella dysenteriae & Ethanol & $\begin{array}{l}\text { Hossain et al., } \\
2012\end{array}$ \\
\hline $\begin{array}{l}\text { Hexane, } \\
\text { Chloroform, } \\
\text { Methanol } \\
\text { (Fruit extract) }\end{array}$ & $\begin{array}{l}\text { E. coli, K. pneumoniae, } P \text {. } \\
\text { vulgaris, M. luteus, B. subtilis, } \\
\text { E. faecalis, S. faecalis }\end{array}$ & $\begin{array}{l}50 \mathrm{mg} / \mathrm{ml} \\
100 \mathrm{mg} / \mathrm{ml}\end{array}$ & $\begin{array}{l}34 \\
36\end{array}$ & $\begin{array}{l}\text { E. faecalis } \\
\text { E. faecalis, K. } \\
\text { pneumoniae }\end{array}$ & Methanol & $\begin{array}{c}\text { Jyothi and Rao, } \\
2011\end{array}$ \\
\hline $\begin{array}{l}\text { Petroleum ether, } \\
\text { Chloroform, } \\
\text { Alcohol } \\
\text { (Fruit extract) }\end{array}$ & $\begin{array}{l}\text { E. coli, } P \text {. aeruginosa, } S \text {. } \\
\text { aeruginosa, } S \text {. aureus, } B \text {. subtilis }\end{array}$ & $\begin{array}{l}10 \mathrm{mg} / \mathrm{ml} \\
20 \mathrm{mg} / \mathrm{ml}\end{array}$ & $\begin{array}{l}12 \\
22\end{array}$ & $\begin{array}{l}\text { S. aureus } \\
\text { S. aureus }\end{array}$ & Alcohol & $\begin{array}{l}\text { Dhale and } \\
\text { Mogle, } 2011\end{array}$ \\
\hline $\begin{array}{l}\text { Methanolic seed } \\
\text { extract }\end{array}$ & $\begin{array}{l}\text { E. coli, S. aureus, } \\
\text { K. pneumoniae, } \\
\text { P. aeruginosa, Enterococcus }\end{array}$ & $\begin{array}{l}50 \mathrm{mg} / \mathrm{ml} \\
100 \mathrm{mg} / \mathrm{ml} \\
150 \mathrm{mg} / \mathrm{ml} \\
200 \mathrm{mg} / \mathrm{ml}\end{array}$ & $\begin{array}{c}14 \\
17 \\
18.5 \\
21\end{array}$ & $\begin{array}{l}\text { P. aeruginosa } \\
\text { E. coli } \\
\text { S. aureus } \\
\text { S. aureus }\end{array}$ & $\begin{array}{l}200 \mathrm{mg} / \mathrm{ml} \text { extract } \\
\text { conc. }\end{array}$ & Priya et al., 20 \\
\hline $\begin{array}{l}\text { Polar flavanoides } \\
\text { (Leaf extract) }\end{array}$ & $\begin{array}{l}\text { P. vulgaris, S. aureus, E. coli, S. } \\
\text { typhi }\end{array}$ & $\begin{array}{l}100 \mathrm{mg} / \mathrm{ml} \\
500 \mathrm{mg} / \mathrm{ml} \\
1000 \mathrm{mg} / \mathrm{ml}\end{array}$ & $\begin{array}{l}17 \\
18 \\
19\end{array}$ & $\begin{array}{c}\text { S. typhi } \\
\text { S. aureus, E. coli, S. } \\
\text { typhi } \\
\text { E. coli, S. typhi }\end{array}$ & $\begin{array}{l}\text { No significant } \\
\text { differences }\end{array}$ & Bansod, 2012 \\
\hline $\begin{array}{l}\text { Non-polar } \\
\text { flavanoides (Leaf } \\
\text { extract) }\end{array}$ & $\begin{array}{l}\text { P. vulgaris, S. aureus, E. coli, S. } \\
\text { typhi }\end{array}$ & $\begin{array}{l}100 \mathrm{mg} / \mathrm{ml} \\
500 \mathrm{mg} / \mathrm{ml} \\
1000 \mathrm{mg} / \mathrm{ml}\end{array}$ & $\begin{array}{l}16 \\
19 \\
19\end{array}$ & $\begin{array}{l}\text { S. aureus, E. coli } \\
\text { E. coli } \\
\text { P. vulgaris }\end{array}$ & & \\
\hline $\begin{array}{l}\text { Tannin (isolated } \\
\text { from leaves of } E \text {. } \\
\text { officinalis) }\end{array}$ & $\begin{array}{l}\text { E. coli, Pseudomonas aeruginosa, } \\
\text { B. subtilis, Shigella boydii, } \\
\text { Shigella flexneri, S. aureus, S. } \\
\text { epidermidis }\end{array}$ & $\begin{array}{c}0.5 \mathrm{mg} / \mathrm{ml} \\
1 \mathrm{mg} / \mathrm{ml} \\
1.5 \mathrm{mg} / \mathrm{ml} \\
2 \mathrm{mg} / \mathrm{ml} \\
2.5 \mathrm{mg} / \mathrm{ml} \\
3 \mathrm{mg} / \mathrm{ml} \\
3.5 \mathrm{mg} / \mathrm{ml} \\
4 \mathrm{mg} / \mathrm{ml} \\
4.5 \mathrm{mg} / \mathrm{ml} \\
5 \mathrm{mg} \cdot \mathrm{ml}\end{array}$ & $\begin{array}{c}\text { Negligible } \\
4.2 \\
8.5 \\
9.5 \\
10.7 \\
11.5 \\
12.9 \\
15.2 \\
17.9 \\
18\end{array}$ & $\begin{array}{c}\text { NA } \\
\text { S. subtilis } \\
\text { E. coli, S. subtilis } \\
\text { E. coli } \\
\text { E. coli } \\
\text { E. coli } \\
\text { E. coli } \\
\text { E. coli } \\
\text { E. coli } \\
\text { E. coli }\end{array}$ & $5 \mathrm{mg} / \mathrm{ml}$ & $\begin{array}{l}\text { Shinde } \text { et al., } \\
2010\end{array}$ \\
\hline $\begin{array}{l}\text { Tannin (isolated } \\
\text { from fruits of } E \text {. } \\
\text { officinalis) }\end{array}$ & $\begin{array}{l}\text { E. coli, Pseudomonas aeruginosa, } \\
\text { B. subtilis, Shigella boydii, } \\
\text { Shigella flexneri, S. aureus, S. } \\
\text { epidermidis }\end{array}$ & $\begin{array}{c}0.5 \mathrm{mg} / \mathrm{ml} \\
1 \mathrm{mg} / \mathrm{ml} \\
1.5 \mathrm{mg} / \mathrm{ml} \\
2 \mathrm{mg} / \mathrm{ml} \\
2.5 \mathrm{mg} / \mathrm{ml} \\
3 \mathrm{mg} / \mathrm{ml} \\
3.5 \mathrm{mg} / \mathrm{ml} \\
4 \mathrm{mg} / \mathrm{ml} \\
4.5 \mathrm{mg} / \mathrm{ml} \\
5 \mathrm{mg} . \mathrm{ml}\end{array}$ & $\begin{array}{c}\text { Negligible } \\
\text { Negligible } \\
2.2 \\
3.1 \\
5.3 \\
6.2 \\
6.8 \\
8.3 \\
8.3 \\
10.1\end{array}$ & $\begin{array}{l}\text { NA } \\
N A \\
\text { E. coli } \\
\text { E. coli } \\
\text { E. coli } \\
\text { E. coli } \\
\text { E. coli } \\
\text { S. boydii } \\
\text { S. boydii } \\
\text { E. coli }\end{array}$ & $5 \mathrm{mg} / \mathrm{ml}$ & $\begin{array}{l}\text { Shinde } \text { et al., } \\
2010\end{array}$ \\
\hline
\end{tabular}

cancer thus demonstrating its potential efficacy for treatment of human papillomavirus-induced cervical cancers (Mahata et al., 2013).

An in vitro cytotoxicity was performed against five human cancer cell lines and the activity was done using $100 \mu \mathrm{g} / \mathrm{ml}$ of the ethanolic whole plant extract of $E$. officinalis. Against lung (A-549) cell line plant extract showed $82 \%$ growth inhibition. In case of liver cell line (Hep-2), it showed no activity, whereas in colon 502713 cell line, the plant extract displayed maximum activity. In case of IMR-32 neuroblastima cell line and HT-29 liver human cancer line, the plant extract showed $97 \%$ and $98 \%$ activity, respectively (Verma et al., 2012). E. officinalis fruit extract at $50-100 \mu \mathrm{g} / \mathrm{mL}$ can significantly inhibit cell growth of six human cancer cell lines, A549 (lung), HepG2 (liver), HeLa (cervical), MDA-MB-231 (breast), SK-OV3 (ovarian) and SW620 (colorectal). (Ngamkitidechakul et al., 2010). HepG2 and A549 cells were treated with P. emblica and $T$. bellerica extracts alone or in combination with doxorubicin or cisplatin and effects on cell growth were determined using the sulforhodamine B (SRB) assay. Both the plant extracts demonstrated growth inhibitory activity against the two cancer cell lines tested (Pinmai et al., 2008). Studies also demonstrated that amla extracts are cytotoxic and restrain the in vitro proliferation of some tumor cell lines such as MK-1 (human gastric adenocarcinoma) and B16F10 (murine melanoma) (Zhang et al., 2004).

HIV-reverse transcriptase inhibitory activity

Inhibition of HIV-Reverse Transcriptase (HIV-RT) by $P$. emblica plant extract fractions was tested on Peripheral Blood Mononuclear Cells. From this test it was observed 
Table 6: Traditional uses of Emblica officinalis.

\begin{tabular}{|c|c|c|c|c|c|}
\hline Used part & Preparation/Administration & Dose & Activity & Treatment & References \\
\hline Fruit & $\begin{array}{l}\text { The fruit or fresh fruit is pickled or pre- } \\
\text { served in sugar. Used when dry. }\end{array}$ & $\begin{array}{l}\text { One or two } \\
\text { fruits daily }\end{array}$ & Laxative & Constipation & $\begin{array}{l}\text { Kumar } \text { et al., 2012b; } \\
\text { Baliga and Dsouza, } \\
2010\end{array}$ \\
\hline $\begin{array}{l}\text { Leaves, fresh } \\
\text { fruit, seed }\end{array}$ & $\begin{array}{l}\text { Decoction of leaves or decoction of seed, } \\
\text { dried grapes and sugar (for gargling) or } \\
\text { decoction of fresh fruit and compounds } \\
\text { containing equal part of Emblica seed, } \\
\text { chitrak root, chebulic myrobalan and pipli } \\
\text { is given. }\end{array}$ & $\begin{array}{l}\text { Not } \\
\text { confirmed }\end{array}$ & $\begin{array}{l}\text { Refrigerant and } \\
\text { aperient }\end{array}$ & Fever & $\begin{array}{l}\text { Kumar et al., 2012b; } \\
\text { Patel and Goyal, } \\
\text { 2011; Srivasuki, } \\
2012\end{array}$ \\
\hline Fruit & $\begin{array}{l}\text { Tablespoon of juice is mixed with a cup of } \\
\text { bitter gourd juice }\end{array}$ & $\begin{array}{l}\text { taken daily } \\
\text { for two } \\
\text { months }\end{array}$ & $\begin{array}{l}\text { Antidiabetic } \\
\text { activity }\end{array}$ & $\begin{array}{l}\text { Diabetes, eye } \\
\text { complication in } \\
\text { diabetes }\end{array}$ & $\begin{array}{l}\text { Kumar et al., 2012a; } \\
\text { Singh et al., } 2011\end{array}$ \\
\hline $\begin{array}{l}\text { Fruit, bark, } \\
\text { root, leaves }\end{array}$ & $\begin{array}{l}\text { Fruit decoction is mixed with sour milk or, } \\
\text { bark partakes of the astringency of the } \\
\text { fruit. Decoction and evaporation of the root } \\
\text { solution produces an astringent extract } \\
\text { equal to catechu. An infusion of the leaves } \\
\text { with fenugreek seed is also given. }\end{array}$ & $\begin{array}{l}\text { Not } \\
\text { confirmed }\end{array}$ & $\begin{array}{l}\text { Anti-diarrheal } \\
\text { activity }\end{array}$ & $\begin{array}{l}\text { Diarrhoea, chronic } \\
\text { diarrhea }\end{array}$ & $\begin{array}{l}\text { Kumar et al., 2012b; } \\
\text { Srivasuki, } 2012\end{array}$ \\
\hline \multirow[t]{3}{*}{$\begin{array}{l}\text { Root, leaves, } \\
\text { node }\end{array}$} & $10 \mathrm{gm}$ roots are taken and ground. & $\begin{array}{l}\text { Taken } \\
\text { twice after } \\
\text { meal per } \\
\text { day. }\end{array}$ & $\begin{array}{l}\text { Pain killing, anti- } \\
\text { inflammatory } \\
\text { activity }\end{array}$ & Dental problems & $\begin{array}{l}\text { Kumar et al., 2012b; } \\
\text { Srivasuki, } 2012\end{array}$ \\
\hline & Leaves are squeezed and the juice extracted & $\begin{array}{l}\text { A few } \\
\text { drops of } \\
\text { juice is put } \\
\text { in the ear }\end{array}$ & & & \\
\hline & $\begin{array}{l}\text { Grind the node and mix it with water. } \\
\text { After vigorous stirring it is filtered through } \\
\text { a cloth. Water drop is given to right ear left } \\
\text { sided teeth are in pain and vice versa. }\end{array}$ & $\begin{array}{l}\text { Only few } \\
\text { drops }\end{array}$ & & & \\
\hline Bark & $\begin{array}{l}\text { The juice of the bark combined with honey } \\
\text { and turmuric is given }\end{array}$ & $\begin{array}{l}\text { Not } \\
\text { confirmed }\end{array}$ & $\begin{array}{l}\text { Antimicrobial } \\
\text { activity }\end{array}$ & Gonorrhoea & $\begin{array}{l}\text { Kumar et al., 2012b; } \\
\text { Srivasuki, } 2012\end{array}$ \\
\hline Fruit & Fresh fruits or crushed fruits & $\begin{array}{l}\text { Not } \\
\text { confirmed }\end{array}$ & $\begin{array}{l}\text { Growth promoting } \\
\text { effects }\end{array}$ & Hair growth & $\begin{array}{l}\text { Singh et al., 2011; } \\
\text { Patel and Goyal, } \\
\text { 2011; }\end{array}$ \\
\hline Fruit & A paste of the fruit is a useful application to & $\begin{array}{l}\text { Not } \\
\text { confirmed }\end{array}$ & $\begin{array}{l}\text { Headache, nausea } \\
\text { or vomiting } \\
\text { inhibitory effect }\end{array}$ & $\begin{array}{l}\text { Cephalalgia } \\
\text { (headache) }\end{array}$ & $\begin{array}{l}\text { Kumar et al., 2012b, } \\
\text { Patel and Goyal, } \\
2011\end{array}$ \\
\hline $\begin{array}{l}\text { Leaves, root } \\
\text { bark }\end{array}$ & $\begin{array}{l}\text { Decoction of the leaves or root bark mixed } \\
\text { with honey is applied to inflammations of } \\
\text { the mouth }\end{array}$ & $\begin{array}{l}\text { Not } \\
\text { confirmed }\end{array}$ & $\begin{array}{l}\text { Anti-inflammatory, } \\
\text { bactericidal activity }\end{array}$ & $\begin{array}{l}\text { Treatment of } \\
\text { aphthae or } \\
\text { aphthous stomati- } \\
\text { tis }\end{array}$ & Kumar et al., 2012b \\
\hline Fruit & $\begin{array}{l}\text { One teaspoonful of powder of the dry fruit } \\
\text { mixed with two teaspoon full of jaggery }\end{array}$ & $\begin{array}{l}\text { Taken } \\
\text { twice daily } \\
\text { for a } \\
\text { month }\end{array}$ & $\begin{array}{l}\text { Anti-rheumatic } \\
\text { activity }\end{array}$ & Rheumatism & Kumar et al., 2012a \\
\hline
\end{tabular}

that aqueous fraction and n-hexane fraction have highest inhibition of recombinant HIV-RT $(91 \%$ and $89 \%$, respectively) at $1 \mathrm{mg} / \mathrm{ml}$ concentration. Chloroform fraction showed highest inhibition of HIV-RT at $0.5 \mathrm{mg} / \mathrm{ml}$ and carbon tetrachloride fraction at $0.12 \mathrm{mg} / \mathrm{ml}$ concentration. At $0.12 \mathrm{mg} / \mathrm{ml}$ and 0.5 concentrations $50 \%$ of the HIVRT activity is inhibited in n-hexane fraction and carbon tetrachloride fraction respectively (Estari et al., 2012).

\section{Anti ulcerogenic activity}

The ethanolic extract of E. officinalis has found highly effective in controlling growth of $H$. pylori in-vitro with minimum inhibitory control ranging from 0.91 to $1.87 \mu \mathrm{g} /$ $\mu \mathrm{l}$. The result concluded that the plant ethanolic extract is well retained with total phenolics, reducing power, flavanoids and the antioxidant properties which make amla a proper remedial use against $H$. pylori infection and gastric ulcer (Mehrotra et al., 2011).

\section{Antimutagenic and wound healing activity}

An investigation on Swiss albino mice showed that 50\% methanolic extract of Emblica fruit can protect mice against the chromosome damaging effects of the wellknown mutagen cyclophosphamide (Agrawal et al., 2012). Ascorbic acid and tannins of E. officinalis, namely emblicanin A and emblicanin B have strong antioxidant action and it is proposed that the addition of these antioxidants support the repair process of cells. Emblica 
increases cellular proliferation at the wound site, as supported by a raise in the action of extracellular signalregulated kinase $1 / 2$, along with an increase in DNA, type III collagen, acid-soluble collagen, aldehyde content, shrinkage temperature and tensile strength (Sumitra et al., 2009).

\section{In vitro propagation}

A simple and one step reproducible protocol was developed by Thilaga et al. (2013) for induction of high frequency somatic embryogenesis from juvenile leaf tissues of Emblica officinalis in vitro. Highest percentage of callus $(67.5 \%)$ was obtained on media containing $0.45 \mu \mathrm{M}$ 2 , 4-dichlorophenoxyacetic in combination with $22 \mu \mathrm{M}$ 6benzylaminopurine. Somatic embryogenesis and plantlet regeneration of Emblica officinalis was performed by using in vitro germinated seeds derived cotyledon explants to produce proembryos directly in MS media (Al-Sabah et al., 2012). Another efficient protocol for in vitro shoot proliferation of Emblica officinalis has been evaluated by using nodal explants where MS medium was found the best for shoot proliferation (Goyal and Bhadauria, 2007)

\section{TRADITIONAL USES}

Traditionally E. officinalis have been used for the ailments of different diseases in different countries for ancient periods. Traditional uses of E. officinalis are summarized in table 6 .

\section{CONCLUSION}

Amla or Indian gooseberry has been playing a significant role from ancient times in traditional medicine, Ayurveda and in tribal medicine. The major group of phytochemicals of like tannins, flavonoids, terpenoids, tannins and other polyphenolic compounds extracted from Amla has been screened for diverse biological and biopharmaceutical investigations from last few decades. Some important Amla phytochemicals like gallic acid, ellagic acid, emblicanin A, emblacani B, quercetin, phyllantine, phyllantidine and so forth have been confirmed as having different biological activities like antioxidant, antimicrobial, anti-inflammatory, antidiabetic, antitissuive, anti, radioprotective, chemopreventive, wound healing activities and so on. From the current investigation, it has seen that some bioactive compounds from Emblica officinalis are also common in other medicinal plant species. These phytochemicals extracted from other plants has been investigated for different bioscreening showing significant results but have not been researched from Emblica officinalis solvent extraction yet. Therefore, further evaluation of unexplored bioactive compounds of Amla, is needed which can reveal more and more new biological activities of this potent medicinal plant.

\section{REFERENCES}

Agrawal RC, Sharma R and S.k. M (2012). Antimutagenic and wound healing activity of Emblica officinalis extract in Swiss Albino mice. Int. J. Sci. \& Eng. Res. 3(5): 1-12.

Al-Sabah L, Sudhersan C and Manuel SJ (2012). Somatic Embryogenesis and Plantlet Regeneration in Amla. Am.-Eurasian J. Sustain. Agric. 6(4): 417-42.

Baliga MS and Dsouza JJ. 2010. Amla (Emblica officinalis Gaertn), a wonder berry in the treatment and prevention of cancer. Euro. J. of Cancer Prev. 20: 225-239. [DOI]

Bansod KD (2012). Isolation and study of antimicrobial activities of polar and non-polar flavanoids from the leaves of Phyllanthus emblica. Der Pharma Chemica. 4(5): 1833-1835.
Beutler JA, Karbon EW, Brubaker AN, Malik R, Curtis DR, Enna SJ (1985). Securinine alkaloids: a new class of GABA receptor antagonist. Brain Res. 330(1): 135-140. [DOI]

Bhattacharya A, Chatterjee A, Ghosal S and Bhattacharya SK (1999). Antioxidant activity of active tannoid principles of Emblica officinalis (Amla). Indian J. Exp. Biol. 37: 676-680. PMid:10522157

Bobbarala V, Katikala PK, Naidu KC and Penumajji S (2009). Antifungal activity of selected plant extracts against phytopathogenic fungi Aspergillus niger F2723. Indian J.Sci.Technol. 2(4): 87-90.

Chaieb I (2010). Saponins as insecticides: a review. Tunisian J. Plant Prot. 5 39-50.

Chang JH, Cho JH, Kim HH, Lee KP, Lee MW, Han SS and Lee DI (1995). Antitumor activity of pedunculagin, one of the ellagitannin. Arch. of Pharmacal Res. 18(6): 396-401. [DOI]

Charoenteeraboon J, Ngamkitidechakul C, Soonthornchareonnon N, Jaijoy $\mathrm{K}$ and Sireeratawong S (2010). Antioxidant activities of the standardized water extract from fruit of Phyllanthus emblica Linn. Songklanakarin J. Sci. Technol. 32 (6): 599-604.

Chen PS and Li JH (2006). Chemopreventive effect of punicalagin, a novel tannin component isolated from Terminalia catappa, on H-rastransformed NIH3T3 cells. Toxicology Letters. 163(1): 44-53. [DOI]

Deep P, Murugananthan G and Nandkumar (2011). Herbal formulation and its evaluation for antidiabetic activity. Pharmacologyonline. 3 : 1134-1144.

Dhale DA and Mogle UP (2011). Phytochemical screening and antibacterial activity of Phyllanthus emblica (L.). Sci. Res. Rep. 1(3): 138 -142.

Estari M, Venkanna L, Sripriya D and Lalitha R (2012). Human Immunodeficiency Virus (HIV-1) reverse transcriptase inhibitory activity of Phyllanthus emblica plant extract. Biol. Med. 4 (4): 178-182.

Gopa B, Bhatt J and Hemavathi KG (2012). A comparative clinical study of hypolipidemic efficacy of Amla (Emblica officinalis) with 3-hydroxy-3methylglutaryl-coenzyme-A reductase inhibitor simvastatin. Indian J Pharmacol. 44(2): 238-242. [DOI]

Goyal D and Bhadauria S (2008). In vitro shoot proliferation in Emblica officinalis var. Balwant from nodal explants. Ind. J. Biotechnol. 7: 394397.

Hazra B, Sarkar R, Biswas S and Mandal N (2010). Comparative study of the antioxidant and reactive oxygen species scavenging properties in the extracts of the fruits of Terminalia chebula, Terminalia belerica and Emblica officinalis. BMC Complementary and Alternative Med. 10: 1-15. [DOI]

Hossain MM, Mazumder K, Hossen SMM, Tanmy TT and Rashid M] (2012). In vitro studies on antibacterial and antifungal activities of Emblica officinalis. Int. J. Pharm. Sci. Res. 3(4): 1124-1127.

Jagetia GC, Baliga MS, Malagi KJ and Kamath MS (2002). The evaluation of the radioprotective effect of Triphala (an ayurvedic rejuvenating drug) in the mice exposed to $\gamma$-radiation. Phytomedicine. 9: 99-108. [DOI]

Jaijoy K, Soonthornchareonnon N, Panthong A and Sireeratawong S (2010). Anti-inflammatory and analgesic activities of the water extract from the fruit of Phyllanthus emblica Linn. Int. J. App. Res. Nat. Prod. 3 (2): 28-35.

Jeyasankar A, Premalatha and Elumalai K (2012). Larvicidal activity of Phyllanthus emblica Linn. (Euphorbiaceae) leaf extracts against important human vector mosquitoes (Diptera: Culicidae). Asian Pacific J. Trop. Dis. 1(2): 399-403. [DOI]

Jyothi S and Rao BS (2011). Screening of antibacterial activity of Emblica officinalis L. fruits. Pharmacologyonline. 3: 848-852.

Karamaæ M, Kosiñska A and Pegg RB (2006). Content of gallic acid in selected plant extracts. Pol. J. Food Nutr. Sci. 15/56(1): 55-58

Khan KH (2009). Roles of Emblica officinalis in Medicine - A Review. Bot. Res. Int. 2(4): 218-228.

Kumar A, Singh A and Dora J (2012a). Essential perspectives for Emblica offcinalis. Int. J. Pharma. Chem. Sci. 1(1): 11-18.

Kumar CS and Kalaivani R (2011). Hypolipedemic effect of Emblica officinalis on histopathological study and DNA fragmentation analysis in experimentally induced hypercholesteremic rats. Int. J. Pharma Sci. Res. 2(8): 168-175.

Kumar KPS, Bhowmik D, Dutta A, Yadav AP, Paswan S, Srivastava S and Deb L (2012b). Recent Trends in Potential Traditional Indian Herbs Emblica officinalis and Its Medicinal Importance. J. Pharmacog. and Phytochem. 1(1): 24-32.

Kumar VN, Vibha and Ashwani K (2013). A Comparative study of heavy metals in Emblica officinalis, Phyllanthus emblica and Azadirachta indica. Int. Res. J. Biological Sci. 2(8): 16-19.

Liu X, Cui C, Zhao M, Wang J, Luo W, Yang B and Jiang Y (2008a) Identification of phenolics in the fruit of emblica (Phyllanthus emblica L.) and their antioxidant activities. Food Chem. 109: 909-915. [DOI] 
Liu X, Zhao M, Wang J, Yang B and Jiang Y (2008b). Antioxidant activity of methanolic extract of emblica fruit (Phyllanthus emblica L.) from six regions in China. J. Food Composition and Analysis. 21: 219-228. [DOI]

Madhuri S, Pandey G and Verma KS (2011). Antioxidant, immunomodulatory and anticancer activities of Emblica officinalis: an overview. Int. Res. J. Pharm. 2(8): 38-42.

Mahata S, Pandey A, Shukla S, Tyagi A, Husain SA, Das BC and Bharti AC (2013). Anticancer Activity of Phyllanthus emblica Linn. (Indian Gooseberry): Inhibition of Transcription Factor AP-1 and HPV Gene Expression in Cervical Cancer Cells. Nutrition and Cancer. 65(1): 88-97. [DOI]

Majumdar S, Bhattacharya S and Haldar PK (2010). Comparative in vitro free radical scavenging activity of some indigenous plants. Int. J. PharmTech Res. 2(2): 1046-1049.

Malar HLV and Bai SMM (2009). Hepato-protective activity of Phyllanthus emblica against paracetamol induced hepatic damage in Wister Albino rats. Afr. J. Basic \& Applied Sci. 1(1-2): 21-25.

Meena AK, Singh A and Rao MM (2010). Evaluation of physicochemical and preliminary phytochemical studies on the fruit of Emblica officinalis Gaertn. Asian J. Pharma. and Clin. Res. 3(3): 242-243.

Mehrotra S, Jamwal R, Shyam R, Meena DK, Mishra K, Patra R, De R, Mukhopadhyay A, Srivastava AK and Nandi SP (2011). AntiHelicobacter pyloriand antioxidant properties of Emblica officinalis pulp extract: A potential source for therapeutic use against gastric ulcer. J. Med. Plant. Res. 5(12): 2577-2583.

Mir AI, Kumar B, Tasduq SA, Gupta DK, Bhardwaj S and Johri RK (2007). Reversal of hepatotoxin-induced pre-fibrogenic events by Emblica officinalis- A historical study. Ind. J. Exp. Biol. 45: 626-629. PMid:17821859

Mishra V, Agrawal M, Onasanwo SA, Mdhur G, Rastogi P, Pandey HP Palit G and Narender T (2013). Anti-secretory and cyto-protective effects of chebulinic acid isolated from the fruits of Terminalia chebula on gastric ulcers. Phytomedicine. 20(6): 506-511. [DOI]

Modilal MRD and Pitchai D (2011). Hypoglycemic and hypolipidemic effects of Phyllanthus (Euphorbiaceae) fruits in alloxan-induced diabetic rats. J. Biotech. and Biotherapeutics. 1(5): 34-39.

Murugan K, Madhiyazhagan P, Nareshkumar A, nataraj T, Dinesh D, Hwang JS and Nicoletti M (2012). Mosquitocidal and water purification properties of Ocimum sanctum and Phyllanthus emblica. J. Entomological and Acarological Res. 44(e17): 90-97.

Muthuraman A, Sood S and Singla SK (2011). The antiinflammatory potential of phenolic compoundsfrom Emblica officinalis L. in rat. Inflammopharmacol. 19:327-334. [DOI]

Negi AS, Darokar MP, Chattopadhyay SK, Garg A, Bhattacharya AK, Srivastava V and Khanuja SPS (2005). Synthesis of a novel plant growth promoter from gallic acid. Bioorg. Med. Chem. Lett. 15: 1243-1247. [DOI]

Ngamkitidechakul C, Jaijoy K, Hansakul P, Soonthornchareonnon N and Sireeratawong S (2010). Antitumour effects of Phyllanthus emblica L.: Induction of cancer cell apoptosis and inhibition of in vivo tumour promotion and in vitro invasion of human cancer cells. Phytother. Res. 24: 1405-1413. [DOI]

O"zer O, Mutlu B and $\mathrm{K}_{1 v c_{3}}$ ak B (2007). Antityrosinase activity of some plant extracts and formulations containing ellagic acid. Pharma. Bio. 45(6): 519-524. [DOI]

Papoutsi Z, Kassi E, Tsiapara A, Fokialakis N, Chrousos GP and Moutsatsou P (2005). Evaluation of estrogenic/antiestrogenic activity of ellagic acid via the estrogen receptor subtypes ER $\alpha$ and ER $\beta$. J. Agric. Food Chem. 53:7715-7720. [DOI]

Patel SS and Goyal RK (2011). Emblica officinalis Geartn.: A Comprehensive review on Phytochemistry, Pharmacology and Ethnomedicinal Uses. Res. J. Med. Plant, 2011. 1-11.

Pemminati S, H.N G, Shenoy AK, Sahu SS, Mishra S, Meti V and Vinod N (2010). Antidepressant activity of aqueous extract of fruits of Emblica officinalis in mice. Int. J. App. Biol. Pharma. Technol. 1(2): 449-454.

Phetkate P, Kummalue T, U-pratya Y and Kietinun S (2012). Significant increase in Cytotoxic T Lymphocytes and Natural Killer cells by Triphala: A clinical phase I study. Evidence-Based Complementary and Alternative Medicine. Volume 2012: 1-6. [DOI]

Pinmai K, Chunlaratthanabhorn S, Ngamkitidechakul C, Soonthornchareon N and Hahnvajanawong C (2008). Synergistic growth inhibitory effects of Phyllanthus emblica and Terminalia bellerica extracts with conventional cytotoxic agents: doxorubicin and cisplatin against human hepatocellular carcinoma and lung cancer cells. World J. Gastroenterol. 14(10): 1491-1497. [DOI]
Prakash D, Upadhyay G, Gupta C, Pushpangadan P and Singh KK (2012). Antioxidant and free radical scavenging activities of some promising wild edible fruits. Int. Food Res. J. 19 (3): 1109-1116.

Priya G, Parminder N and Jaspreet S (2012). Antimicrobial and antioxidant activity on Emblica officinalis seed extract. Int. J. Res. Ayur. Pharma. 3(4) 591-596.

Qureshi SA, Asad W and Sultana V (2009). The effect of Phyllanthus emblica Linn on type - II diabetes, triglycerides and liver - specific enzyme. Pak. J. Nutri. 8 (2): 125-128. [DOI]

Rahman S, Akbor MM, Howlader A and Jabbar A (2009). Antimicrobial and cytotoxic activity of the Alkaloids of Amlaki (Emblica officinalis). Pak. J. Biol. Sci. 12: 1152-1155. [DOI]

Rai N, Tiwari L, Sharma RK and Verma AK (2012). Pharmaco-botanical Profile on Emblica officinalis Gaertn. - A Pharmacopoeial Herbal Drug. STM Journals. 1(1): 29-41.

Reddy DB, Reddy TCM, Jyotsna G, Sharan S, Priya N, Lakshmipathi V and Reddanna P (2009). Chebulagic acid, a COX-LOX dual inhibitor isolated from the fruits of Terminalia chebula Retz., induces apoptosis in COLO-205 cell line. J. Ethnopharmacol. 124: 506-512. [DOI]

Santoshkumar J, Manjunath S and Sakhare PM (2013). A study of antihyperlipedemia, hypolipedemic and anti-atherogenic activity of fruit of Emblica officinalis (amla) in high fat fed Albino rats. Int. J. Med. Res. Health Sci. 2(1): 70-77.

Satish S, Mohana DC, Raghavendra MP and Raveesha KA (2007). Antifungal activity of some plant extracts against important seed borne pathogens of Aspergillus sp. J. Agri. Tech. 3(1): 109-119.

Satyanarayana T, Reddy PD, Swarnalatha D and Mathews AA (2010). Hypoglycemic effect of a poly herbal extract on normal and streptozotocin induced diabetic rats. Int. J. Pharm. Pharma. Sci. 2(3): 56-57.

Sharma RJ, Chaphalkar SR and Adsool AD (2010). Evaluating antioxidant potential, cytotoxicity and intestinal absorption of flavonoids extracted from medicinal plants. Int. J. Biotechnol. App. 2(1): 01-05.

Shinde SL, Junne SB, Shinde AT, Patil SA and Wadje SS (2010). Antibacterial properties of tannins isolated from leaves and fruits of Emblica officinalis Gaertn. Res. J. Pharma. Biol. Chem. Sci. 1(3): 699-703.

Shivaji BB, Manju R, Nagaraj M, Sandhya V, Supriya G, Pranitha K, Kiran $B$ and Lalitha V (2010). Comparative study of antibacterial and antioxidant activity of plant extract- Amla [Phyllanthus emblica L.] Tulsi [Ocimum tenuiflorum L.] Neem [Azadirachta indica A.JUSS]. Pharmacophore. 1(3): 178-183.

Singh E, Sharma S, Pareek A, Dwivedi J, Yadav S and Sharma S (2011). Phytochemistry, traditional uses and cancer chemopreventive activity of Amla (Phyllanthus emblica): the sustainer. J. App. Pharma. Sci. 2 (1) 176-183.

Singh I, Sharma A, Jindal A, Soyal D and Goyal PK (2006). Protective effect of Emblica officinalis fruit extract against gamma irradiation in mice. Pharmacologyonline. 2: 128-150.

Srikumar R, Parthasarathy NJ and Sheela DR (2005). Immunomodulatory activity of triphala on neutrophil functions. Biol. Pharm. Bull. 28(8): 1398-403. [DOI]

Suja RS, Nair AMC, Sujith S, Preethy J and Deepa AK (2009). Evaluation of immunomodulatory potential of Emblica officinalis fruit pulp extract in mice. Indian J. Anim. Res. 43(2): 103-106.

Sumitra M, Manikandan P, Gayathri VS, Mahendran P and Suguna L (2009). Emblica officinalis exerts wound healing action through upregulation of collagen and extracellular signal-regulated kinases (ERK1/2). Wound Repair Regen. 17(1): 99-107. [DOI]

Thilaga S, Largia MJV, Parameswari A, Nair RR and Ganesh D (2013). High frequency somatic embryogenesis from leaf tissue of Emblica officinalis Gaertn. - A high valued tree for non-timber forest products. Aus. J. Crop Sci. 7(10):1480-1487.

Vazirian M, Khanavi M, Amanzadeh Y and Hajimehdipoor H (2011). Quantification of galic acid in fruits of three medicinal plants. Int. J. Pharmaceutical Res. 10(2): 233-236.

Verma SK, Shaban A, Nautiyal R, Purohit R, Singh S and Chimata ML (2012). In vitro cytotoxicity of Emblica officinalis against different human cancer cell lines. Asian J. Pharma. \& Clin. Res. 5(2): 77-78.

Zhang YJ, Nagao T, Tanaka T, Yang CR, Okabe H and Kouno I (2004). Antiproliferative Activity of the Main Constituents from Phyllanthus emblica. Biol. Pharm. Bull. 27(2): 251-255. [DOI] 